\title{
Address on Celestial Photography
}

(With Lanteru Illustrations).

By Mr Alex. Morgan, M.A., B.Sc.

Second Meeting, December 13th, 1895.

Wyr. Peddie, Esq., D.Sc., F.R.S.E., President, in the Chair.

Note on the Circles of Curvature of a Plane Curve.

By Professor Tait.

When the curvature of a plane curve continuously increases or diminishes (as is the case with a logarithmic spiral, for instance) 110 two of its circles of curvature ciln intersect one another.

This curious remark occurred to me some time ago in connection with an accidental feature of a totally different question. (Systems of Plane Curves whose Orthoyonals form a Similar System. Proc. R.S.E., $6 / 5 / 95$.

The proof is excessively simple. For, if $\mathbf{A}, \mathbf{b}$, be any two points of the evolute, the chord $A B$ is the clistance between the centres of two of the circles, and is necessarily less than the arc $A B$, the difference of their radii. Thus one of the circles lies wholly within the other. (This is true even if the evolute be sinuous, so that the original curve has ramphoid cusps.)

When the curve has points of maximum or minimum curvature, there are corresponding cusps on the evolute ; and pairs of circles of curvature, whose centres lie on opposite sides of the cusp, C, may intersect:- - for the chord $A B$ may now exceed the difference between C.A and CI: 